\title{
PRESENTACIÓN
}

\section{FILOSOFÍA Y ESTILOS DE VIDA PARA NUESTRO PRESENTE}

\author{
Mario Germán Gil \\ Grupo Redipe: Educación, Epistemología y \\ diversidad
}

El título del presente número de la Revista Boletín Redipe reivindica la relación inescindible entre Filosofía y vida. Particularmente los tres primeros artículos, incluido el editorial, aportan al esclarecimiento del nexo entre filosofía y estilos de vida para nuestro presente. Se destaca el esfuerzo de tres miradas en torno al asunto, asumidas por tres estudiosos de la filosofía interesados por la temática. ¿Qué papel juega hoy la filosofía para tiempos tan complejos como los que afrontamos y cómo incide en los estilos de vida? Los escritos recogen reflexiones que giran alrededor de esta pregunta. El primero, Las artes de la existencia en los modos de ser. Una lectura a cuatro textos de Agamben, recoge un llamado que aborda el significado de la filosofía y su impacto en los modos de ser contemporáneo. El segundo, De Sócrates a Foucault: una larga tradición de la filosofía como forma de vida y arte de vivir a la luz de Pierre Hadot y Alexander Nehamas, traza un arco histórico desde Sócrates a Foucault, pasando por Epicteto, según el cual el hilo conductor de la Filosofía es una forma integral de vida y/o arte de vivir y no una simple actividad especulativa académica. El tercero, Gómez Dávila y la filosofía como forma de vida, prepondera la importancia de la filosofía en su sentido práctico, íntimo y singular como compromiso de vida para nuestro momento.

Invitamos al lector a abordar estas lecturas filosóficas, tan necesarias para nuestro presente.

Los demás trabajos que conforman la publicación son, por una parte, la entrevista con Olga Lizasoáin Rumeu sobre pedagogía hospitalaria, realizada por el académico chileno Aldo Ocampo González, Director fundador del Centro de Estudios Latinoamericanos de Educación Inclusiva (CELEI). Y luego están los artículos de investigación, revisión y reflexión en torno al uso utilitarista de la información y el conocimiento, y a diversos ámbitos, campos y tópicos de la educación tales como la pedagogía, el aprendizaje, estrategias didácticas, inclusión social y las Tic, entre otros de relevancia en los procesos que pretende fortalecer este complejo de docentes e instituciones iberoamericanas, Redipe. Veamos. 
EL CONTROL OLIGOPÓLICO DE LA INFORMACIÓNYDELOSENTRETENIMIENTOS: HERRAMIENTAALSERVICIODELAIDEOLOGÍA YCULTURASDOMINANTES. Artículo de reflexión propositiva a cargo de Alejandro Teitelbaum. Miembro Red Iberoamericana de Pedagogía, Lyon, Francia. Subraya qye la base material del control y la manipulación de las conciencias para someterlas a las ideas de las clases dominantes son los monopolios de los grandes medios de comunicación de masas. Con el desarrollo de las tecnologías de la comunicación se han formado grandes conglomerados transnacionales que abarcan la producción y utilización de los soportes materiales: editoriales, periódicos, radiodifusoras, filmes, emisoras de televisión, vídeos, satélites, medios electrónicos, etc., que dominan también las redes de comercialización y difusión. Esas bases materiales sirven para transmitir los mensajes de politólogos, economistas, filósofos, libretistas de programas de TV, programadores de plays-stations, etc., destinados a anestesiar la capacidad reflexiva y crítica del público receptor. Que se complementan con estudios especializados que tienen por objetivo mejorar la penetración del mensaje de la ideología y cultura dominantes.

\section{APLICACIÓN DEL MODELADO EN EL DESARROLLO DE LA CREATIVIDAD EN EL DIBUJO DE NIÑOS PRE-ESQUEMÁTICOS.}

Artículo de investigación del académico chileno Nelson Oyarzún Oyarzún, Universidad de Los Lagos. Presenta la técnica del modelado aplicado al proceso de enseñanza de las artes visuales. Cómo influye y potencia el desarrollo de la creatividad en los dibujos de los niños preesquemáticos, entre las edades de 4 a 6 años. El objetivo de este estudio es demostrar cómo el modelado genera el desarrollo de la creatividad en el dibujo, incorporando críticas que permita mejorar este proceso. El diseño fue experimental, con un grupo control y mediciones "antes" y después", en las variables independiente y dependiente. Los resultados obtenidos indican que en la etapa pre-esquemática cuando se trata de incorporar la técnica del modelado, se produce un avance de la capacidad creadora del niño, especialmente en el dibujo del esquema, concepto espacial y el uso del color. Sin embargo, la mayor incorporación de los elementos mencionados se origina en la figura humana, producto de las acciones propias del modelado. Al observar las clases se reveló que la gran preocupación artística del niño sigue siendo representarse a sí mismo y el mundo que le rodea, a través del juego con las pastas de modelar. La innovación pedagógica del profesor es incorporar actividades y motivaciones en el modelado, que impulsen indirectamente el desarrollo creativo en forma natural, en las etapas del arte infantil. No interfiriendo en la espontaneidad propia de los niños.

\section{ANALIZAR LAS PRÁCTICAS PEDAGÓGICAS} DE LOS DOCENTES DEL GRADO TRANSICIÓN DE LA INSTITUCIÓN EDUCATIVA NORMAL SUPERIOR DE SINCELEJO AÑO 2016 -2017. Artículo de investigación de Maritza Tenorio Troncoso. Normal de Sincelejo, CECAR. Vinculado al un proyecto doctoral en torno al diseño curricular que potencialice el pensamiento crítico. Se fundamenta en el análisis de las prácticas pedagógicas suscitadas por los docentes del grado de transición de la Institución Educativa Normal Superior de Sincelejo. En ese sentido, se dispone de un diseño cualitativo, enmarcado en el discurso de un método hermenéutico, dado que se entra en la necesidad de interpretar el rol docente frente a una necesidad de desarrollar un pensamiento crítico en los estudiantes a través de las competencias y coadyuvar a su desarrollo integral. El análisis parte de la codificación y categorización facilitada por el programa ATLAS-ti, permitiéndose realizar las conclusiones que ponen de manifiesto las reflexiones sobre los procesos de la práctica pedagógica, los elementos interrelacionados 
desde las orientaciones inherentes en la malla curricular y el método propio de cada docente.

\section{ANÁLISIS DE LAS PUBLICACIONES SOBRE EL SÍNDROME DE BURNOUT EN EL PROFESORADO ESPAÑOL EN LOS ÚLTIMOS AÑOS (2007-2017). Artículo de} reflexión propositiva elaborado por la colega española Nieves Gutiérrez Ángel. Universidad de Almería. Manifiesta que son diversas las publicaciones relacionadas con el burnout que intentan explicar tanto el significado, como las consecuencias, síntomas, o factores que se presentan asociados a tal síndrome. El presente trabajo realiza una revisión sistemática acerca de aquellas publicaciones relacionadas con el síndrome de burnout en el profesorado español en las distintas etapas educativas. Encontrando una mayor prevalencia del mismo en la etapa de educación secundaria, y una mínima existencia en la etapa de educación infantil. Identificando como factores de riesgo las conductas problemáticas o los conflictos, o aspectos propios del contexto docente. $Y$ como factores protectores, como la Inteligencia Emocional o la autoeficacia.

\section{¿COLOMBIA RESPONDE A LAS EXIGENCIAS} DE LA CALIDAD EN LA EDUCACIÓN SUPERIOR CÓMO PILAR DE CRECIMIENTO?

Artículo de revisión a cargo de la académica Cecilia Garzón Daza, Fundación San Mateo Universidad Católica de Colombia. Manifiesta que dentro del ámbito universitario la calidad ha sido vista con la rigurosidad que la misma requiere, debido a que se ha consolidado como la respuesta positiva a los procesos de acreditación de instituciones y programas universitarios. Razón por la cual, la calidad en la educación superior debe continuar su consolidación en el ámbito universitario con miras a demostrar la capacidad de gestión de la institución universitaria, en pro de favorecer esta instancia en todos los aspectos; en donde, la gestión del conocimiento, investigación e internacionalización van a hacer la respuesta positiva para que Colombia afiance su posicionamiento frente a sus homologas en la región.

\section{INTEGRACIÓNDELASTICENLAENSEÑANZA DE LA HISTORIA EN EDUCACIÓN MEDIA} SUPERIOR. Artículo de investigación elaborado por los mexicanos Oscar David Bustos Torres, Dra. Karla Lariza Parra Encinas. Universidad Autónoma de Baja California. Ponen de presente que las Tecnologías de la Información y la Comunicación se han convertido en el elemento esencial de nuevos contextos y espacios sociales. Las instituciones educativas se encuentran analizando las constantes transformaciones derivadas de la incorporación de las tecnologías en los escenarios educativos actuales. Para lograr particularmente el interés de estudiantes de bachillerato en asignaturas como "Historia", es necesario que el desarrollo de las clases y los programas educativos sean atractivos para ellos. En el presente trabajo se comparte un estudio cuantitativo realizado con los docentes de Historia de una institución de educación media superior pública mexicana, basado en un instrumento que analiza las competencias digitales básicas, clasificadas en siete dimensiones.

\section{GOT IT, AND YOU? LET'S SPEAK} TOGETHER! Artículo de investigación a cargo de Lady Carrero Galindo, Mélany Rodríguez Cáceres. Universidad Pedagógica Nacional. Presenta los resultados obtenidos de una investigación acción que intentó desarrollar habilidades de habla en lengua inglesa con estudiantes de cuarto grado de la institución educativa Liceo Femenino Mercedes Nariño, a través de fábulas digitalizadas y recursos multimedia como estrategia innovadora en la enseñanza de inglés como lengua extranjera. La información se recogió a través de diarios de campo realizados en cada sesión, grabaciones de audio-video y encuestas a las estudiantes 
al culminar la presentación de las fabulas digitalizadas. Posteriormente, los resultados obtenidos revelaron que a pesar de los errores orales de las estudiantes, ellas lograron interactuar y producir oraciones que iban más allá de la repetición en clase. Además, sus actitudes fueron positivas frente al aprendizaje del inglés gracias al material implementado en la propuesta.

INCLUSIÓN SOCIAL EN LA ATENCIÓN PSICOSOCIAL A VÍCTIMAS EN LOS PROGRAMAS SOCIOEDUCATIVOS $Y$ FAMILIARES EN CONTEXTOS DE PAZ. Artículo de investigación a cargo de Clara Judith Brito Carrillo, Ana Rita Villa Navas y Cielo Caicedo Manjarres, Universidad de la Guajira. Constituye una respuesta a las múltiples situaciones conflictivas evidenciadas en los sectores vulnerables de la sociedad; así mismo, es producto de la investigación titulada: impacto del programa de inclusión social en la atención psicosocial a víctimas en la troncal del caribe del departamento de La Guajira, convirtiéndose en un reto de oportunidades y posibilidades para las poblaciones asentada en una zona geoestratégicas de gran relevancia a nivel económico, político, social y cultural del departamento de La Guajira. 